\title{
Dark resonances in the field of frequency shifted feedback laser radiation
}

\author{
V I Romanenko ${ }^{1}$, A V Romanenko ${ }^{1,2}$, L P Yatsenko ${ }^{1}$, \\ G A Kazakov ${ }^{3}$, A N Litvinov ${ }^{3}$, B G Matisov ${ }^{3}$ and \\ Yu V Rozhdestvensky ${ }^{4}$ \\ ${ }^{1}$ Institute of Physics, Nat. Acad. Sci. of Ukraine, 46, Nauky Ave., Kyiv 03028, \\ Ukraine \\ E-mail: vr@iop.kiev.ua \\ ${ }^{2}$ Kyiv National Taras Shevchenko University 2, Academician Glushkov Ave, Kyiv \\ 03022, Ukraine \\ 3 St. Petersburg State Polytechnical University, 29, Polytechnicheskaya st, St. \\ Petersburg 195251, Russia \\ E-mail: andrey.litvinov@mail.ru \\ ${ }^{4}$ S. I. Vavilov State Optical Institute 12, Birzhevaya Liniya st, St. Petersburg \\ 199034, Russia
}

\begin{abstract}
.
We present a theory of dark resonances in a fluorescence of a three-level atom gas interacting with a polychromatic field of a frequency shifted feedback (FSF) laser. We show that conditions for the resonance observation are optimal when the phase relations between the laser spectral components provide generation of a light pulses train. We study analytically the field broadening and the light shift of the resonances.

PACS numbers: 42.50.Gy, 32.80.Wr
\end{abstract}

Submitted to: J. Phys. B: At. Mol. Opt. Phys.

\section{Introduction}

Investigations of the coherent population trapping (CPT) effect started at the seventies of the last century [1 5 brought to the discovery of a number of related effects and methods such as the electromagnetically-induced transparency effect [6] 9], the stimulated Raman adiabatic passage (STIRAP) [10 13], new methods of the laser cooling [14], and creation of the miniaturized quantum frequency standards [15]. The CPT effect can be used also for the magnetic field measurements [16, 17].

In the simplest case, the CPT effect appears when a 3-level atom with two longlived and one short-lived states interacts with the bichromatic laser radiation, and each spectral component of this radiation excites the transition between the long-lived and 
short-lived states (the $\Lambda$-scheme of the atom-field interaction). In this case, there is a dip (dark resonance) in the fluorescence intensity dependence on the difference frequency between the two spectral components when this frequency difference is close to the transition frequency between the long-lived states. The CPT effect is based on the atom transition in a "coherent dark state", i.e., in the superposition of the two longlived states which does not interact with the laser radiation.

The dark resonance linewidth depends on the duration of the coherent interaction of an atom with the radiation. To increase this time, some amount of a buffer gas can be introduced into the cell with active atoms. The active and buffer atom collision does not practically change the long-lived states coherence whereas the active atom velocity is drastically changed. If the collision rate is high enough, the fast flight of the active atom through the laser beams is changed by the slow diffusion. Therefore, the duration of coherent atom-field interaction increases and the resonance linewidth decreases. Moreover, the deceleration of atomic movement along the laser light propagation direction leads to the decreasing of a Doppler broadening of transition between the two long-lived states (Dicke narrowing [18]). In a buffered cell with rubidium active atoms, the dark resonances of 20-30 Hz linewidth have been observed [19, 20].

Nowadays, there is a growing interest to the dark resonances created by the frequency comb radiation [21 25]. The dark resonance appears when the frequency of the transition between the long-lived states is divisible by the frequency difference of the adjacent field components. In the recent paper [25], the dark resonances were observed in the field of the femtosecond laser radiation. In this case, the pulse repetition frequency sets the frequency difference between the nearby spectral field components. The electromagnetically induced transparency resonances (based on the CPT effect) were observed in a sequence of picosecond pulses produced by a mode-locked diode laser in [22]. The dark resonances of near-100\% contrast was observed in the field of a free running multimode laser interacting with sodium vapors [21]. If both the frequency difference between the two nearby frequency components and the atomic polarization relaxation rate caused by collisions with the buffer gas are much smaller than the Doppler width of the optical transitions, the number of atoms contributing into the dark resonance and, therefore, the dark resonance contrast increases due to a large number of the polychromatic field frequency components.

The frequency shifted feedback (FSF) laser is one of the sources of a frequency comb radiation [26]. The simplicity of the FSF laser radiation parameters control makes these lasers very attractive for the dark resonances investigation. The acousto-optic modulator (AOM) sets the frequency difference between the two nearby spectral components and the resonator length together with the modulation frequency sets their phases.

The dependence of the spectral component phase on its sequence number is quadratic in FSF lasers whereas it is linear in mode-locked lasers [26]. The spectral component phases accounting is required in the description of the atom-comb interaction because an atom can interact with several spectral components simultaneously (see figure 1). 
In the present article we consider the behavior of the dark resonances created by the field of the FSF laser radiation. We find how the dark resonance characteristics depend on the AOM frequency and on the resonator length. For the simplicity, we consider the interaction of a three-level atom with the FSF laser field. This model was successfully applied to the description of the interaction of real atoms with polychromatic fields [23]. Obtained results are applicable to the dark resonance analysis both for cells with a buffer gas and without it.

In section 2 we give an expression for the electric field of the FSF laser and present the density matrix equation of the three-level atom in the laser radiation field. In section 3 we derive the expression for the detected signal, i.e., for the part of fluorescence which is responsible for the dark resonance. Then in section 4 and 5 we consider the limiting cases when the optical coherence relaxation rate is large or small in comparison with the Doppler width. Obtained results make it possible to find the spectral component phase relations that are optimal for the dark resonance observation and to analyze the light shift and the field broadening of the dark resonance. The main results of the paper are summarized in section 6 .

\section{Basic equations}

Let us suppose that the laser field interacting with a three-level atom induces the transition $|1\rangle \leftrightarrow|3\rangle$ and $|2\rangle \leftrightarrow|3\rangle$ (see figure 1). States $|1\rangle$ and $|2\rangle$ are long-lived states (one of them can be the stable state) and the atoms can spontaneously decay from state $|3\rangle$ to the long-lived states with light emission. The laser field interacting with the atoms is

$$
E=\sum_{n} E_{n} \cos \left(k_{n} z-\omega_{n} t-\varphi_{n}\right)
$$

where $E_{n}$ and $\omega_{n}$ are the $n$-th spectral component amplitude and frequency respectively, $k_{n}=\omega_{n} / c$. The frequencies of spectral components are equidistant,

$$
\omega_{n}=\omega+\varpi n .
$$

Here the zero component number was chosen in such a way that $\omega_{0}=\omega$ corresponds to the spectral component of maximum intensity.

We use the following model for the dependencies of $E_{n}$ and $\varphi_{n}$ on the spectral component number:

$$
E_{n}=E_{0} \exp \left(-\frac{n^{2}}{n_{0}^{2}}\right), \quad \varphi_{n}=\alpha n^{2}+\beta n+\varphi_{0} .
$$

These expressions are specific for the FSF laser [26]. Variation of $\beta$ is equivalent to the change of the time origin and therefore not affect the atomic density matrix elements evolution. The initial phase $\varphi_{0}$ can be include in the phase of atomic state. Hereinafter we assume $\beta=0, \varphi_{0}=0$. The coefficient $\alpha$ in (3) is determined by the AOM frequency 


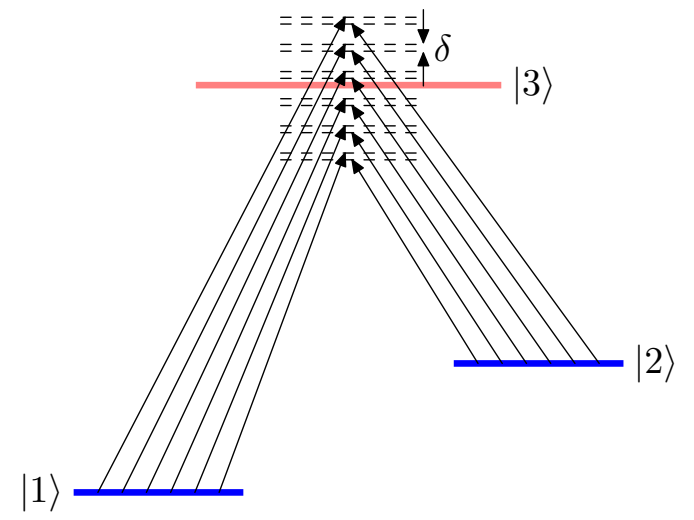

Figure 1. Scheme of interaction between the three-level atom and the polychromatic field with equidistant spectral components

giving the frequency difference $\varpi$ between adjacent spectral components and by the round-trip time $\tau_{r}=L / c$ required for radiation to pass the resonator perimeter $L$,

$$
\alpha=\frac{1}{2} \tau_{r} \varpi .
$$

The equations for the atomic density matrix elements $\rho_{n m}(n, m=1 \ldots 3)$ are

$$
\begin{aligned}
\frac{\mathrm{d}}{\mathrm{d} t} \rho_{n m}= & -\frac{\mathrm{i}}{\hbar} \sum_{l}\left(H_{n l} \rho_{l m}-\rho_{n l} H_{l m}\right) \\
& +\sum_{k l} \Gamma_{n m, k l} \rho_{k l}+(\hat{S} \rho)_{n m},
\end{aligned}
$$

where $\frac{\mathrm{d}}{\mathrm{d} t}=\frac{\partial}{\partial t}+v \frac{\partial}{\partial z}$ is the total time derivative, $v$ is the atomic velocity projection on the laser radiation propagation direction, $H$ is the Hamiltonian of the atom in the laser field, $\Gamma_{n m, k l}$ are the relaxation matrix elements, $\hat{S}$ is the collisional integral.

Let us suppose that the laser field which acts on the atom is small so that the populations $\rho_{i i}, i=1,2,3$, are near to the equilibrium. Then we can solve the set of equations for the density matrix only for the non-diagonal elements supposing that the diagonal elements are in equilibrium. We use the strong collision approximation [27,28] for the collisional term describing the coherence preserving collisions with rate $\nu$ in the equation for $\rho_{12}$. It means that there is no any correlation between the velocity of the atom before and after one collision. Therefore, the equations for the non-diagonal elements of the density matrix read

$$
\begin{aligned}
\frac{\mathrm{d}}{\mathrm{d} t} \rho_{12}= & -\mathrm{i} \sum_{n}\left(\Omega_{1, n} \rho_{32}-\Omega_{2, n} \rho_{13}\right) \\
& \times \cos \left(k_{n} z-\omega_{n} t-\varphi_{n}\right) \\
& +\frac{\mathrm{i}}{\hbar} \omega_{21} \rho_{12}-\gamma_{c o h} \rho_{12} \\
& -\nu\left[\rho_{12}-M(v) N_{12}\right], \\
\frac{\mathrm{d}}{\mathrm{d} t} \rho_{13}= & -\mathrm{i} \sum_{n}\left(\Omega_{1, n}\left[\rho_{33}-\rho_{11}\right]-\Omega_{2, n} \rho_{12}\right)
\end{aligned}
$$


Dark resonances in the field of frequency shifted feedback laser radiation

$$
\begin{aligned}
& \times \cos \left(k_{n} z-\omega_{n} t-\varphi_{n}\right) \\
& +\frac{\mathrm{i}}{\hbar} \omega_{31} \rho_{13}-\gamma^{\prime} \rho_{13}, \\
\frac{\mathrm{d}}{\mathrm{d} t} \rho_{32}= & \mathrm{i} \sum_{n}\left(\Omega_{2, n}\left[\rho_{33}-\rho_{22}\right]-\Omega_{1, n} \rho_{12}\right) \\
& \times \cos \left(k_{n} z-\omega_{n} t-\varphi_{n}\right) \\
& -\frac{\mathrm{i}}{\hbar} \omega_{32} \rho_{32}-\gamma^{\prime} \rho_{32},
\end{aligned}
$$

where $\gamma^{\prime}=\gamma_{c o l}+\frac{1}{2} \gamma_{s p}+\frac{1}{2} \gamma_{L}$ is the $\rho_{13}$ and $\rho_{23}$ matrix elements relaxation rate which depends additively on the collision rate of active atoms with buffer atoms (term $\gamma_{c o l}$ ), on the excited state spontaneous radiation rate $\gamma_{s p}$ and on the laser field frequency fluctuation (term with $\gamma_{L}$ ); the constant $\gamma_{c o h}$ describes the relaxation of the coherence of the long-lived states (density matrix element $\rho_{12}$ );

$$
M(v)=\frac{1}{v_{0} \sqrt{\pi}} \exp \left(-\frac{v^{2}}{v_{0}^{2}}\right)
$$

is the Maxwell distribution, $v_{0}$ is the most probable velocity of the active atom, $\hbar \omega_{n m}$ $(n, m=1,2,3)$ is the difference of the atom energies in $n$ and $m$ states,

$$
N_{12}=\int \rho_{12} d v
$$

The Rabi frequencies

$$
\Omega_{1, n}=-\frac{1}{\hbar} E_{n} d_{13}, \quad \Omega_{2, n}=-\frac{1}{\hbar} E_{n} d_{23},
$$

characterize the interaction of $n$-th spectral field component with the atom. We consider the Rabi frequencies as real because we can assume without loss of generality that the dipole moment matrix elements $d_{13}$ and $d_{23}$ of the transitions between the states $|1\rangle \leftrightarrow|3\rangle$ and $|2\rangle \leftrightarrow|3\rangle$ are real.

The normalization condition for the density matrix has the form

$$
\sum_{j=1}^{3} \int \rho_{j j} d v=1 .
$$

We consider small laser fields $\Omega_{10} \ll \gamma^{\prime}, \Omega_{20} \ll \gamma^{\prime}$, so that the diagonal density matrix elements are close to the equilibrium values. Then we put $\rho_{j j}=p_{j} M(v)$ into equations (77), (8) where $p_{j}$ is a probability for the atom to be in the state $|j\rangle$. Since the population of the excited state is very small $\left(p_{3} \ll p_{1}, p_{2}\right)$ we can neglect $p_{3}$ in comparison with $p_{1}$ and $p_{2}$.

Let us introduce the frequency detunings $\delta_{1}$ and $\delta_{2}$ of the maximum intensity spectral component of the laser field $(n=0$ in equation (2) ) from the frequencies $\omega_{31}$ and $\omega_{32}$ of transitions $|1\rangle \leftrightarrow|3\rangle$ and $|2\rangle \leftrightarrow|3\rangle$ as

$$
\begin{aligned}
& \delta_{1}=\omega_{31}-\omega, \\
& \delta_{2}=\omega_{32}-\omega=\delta_{1}-\omega_{21}
\end{aligned}
$$


Dark resonances in the field of frequency shifted feedback laser radiation

and substitute

$$
\begin{aligned}
& \rho_{13}=\sigma_{13} \exp (-\mathrm{i} k z+\mathrm{i} \omega t), \\
& \rho_{32}=\sigma_{32} \exp (\mathrm{i} k z-\mathrm{i} \omega t) .
\end{aligned}
$$

into (66) -(8), where $k=\omega / c$. In rotating wave approximation we neglect the rapidly oscillating terms $\mathrm{e}^{ \pm 2 \mathrm{i} \omega t}$. Then the equations (66)-(8) read

$$
\begin{aligned}
\frac{\mathrm{d}}{\mathrm{d} t} \rho_{12} & =-\frac{\mathrm{i}}{2} \sum_{n}\left(\Omega_{1, n} \sigma_{32} \mathrm{e}^{\mathrm{i} \Phi_{n}}-\Omega_{2, n} \sigma_{13} \mathrm{e}^{-\mathrm{i} \Phi_{n}}\right) \\
& +\mathrm{i} \omega_{21} \rho_{12}-\gamma_{c o h} \rho_{12}-\nu\left[\rho_{12}-M(v) N_{12}\right], \\
\frac{\mathrm{d}}{\mathrm{d} t} \sigma_{13} & =\frac{\mathrm{i}}{2} \sum_{n}\left[\Omega_{1, n} p_{1} M(v)+\Omega_{2, n} \rho_{12}\right] \mathrm{e}^{\mathrm{i} \Phi_{n}} \\
& +\mathrm{i}\left(\delta_{1}+k v\right) \sigma_{13}-\gamma^{\prime} \sigma_{13}, \\
\frac{\mathrm{d}}{\mathrm{d} t} \sigma_{32} & =-\frac{\mathrm{i}}{2} \sum_{n}\left[\Omega_{2, n} p_{2} M(v)+\Omega_{1, n} \rho_{12}\right] \mathrm{e}^{-\mathrm{i} \Phi_{n}} \\
& -\mathrm{i}\left(\delta_{2}+k v\right) \sigma_{32}-\gamma^{\prime} \sigma_{32},
\end{aligned}
$$

where $\Phi_{n}=n \varpi t+\varphi_{n}-n \kappa z, \kappa=\varpi / c$. We seek for stationary solution of equations (15) -(17) in the form

$$
\begin{aligned}
\rho_{12} & =\sum_{n} r_{12, n} \mathrm{e}^{\mathrm{i} \Phi_{n}}, \\
\sigma_{13} & =\sum_{n} r_{13, n} \mathrm{e}^{\mathrm{i} \Phi_{n}}, \\
\sigma_{32} & =\sum_{n} r_{32, n} \mathrm{e}^{-\mathrm{i} \Phi_{n}},
\end{aligned}
$$

where $r_{l m, n}$ does not depend on time and coordinate.

From the equations (16) and (17), we obtain:

$$
\begin{aligned}
r_{13, n} & =\frac{\mathrm{i} \Omega_{1, n} p_{1} M(v)}{2\left[\gamma^{\prime}-\mathrm{i}\left(\delta_{1}-n \varpi+n \kappa v+k v\right)\right]} \\
& +\sum_{m} \frac{\mathrm{i} \Omega_{2, n-m} \mathrm{e}^{\mathrm{i} \varphi_{m}-\mathrm{i} \varphi_{n}+\mathrm{i} \varphi_{n-m}} r_{12, m}}{2\left[\gamma^{\prime}-\mathrm{i}\left(\delta_{1}-n \varpi+n \kappa v+k v\right)\right]}, \\
r_{32, n} & =-\frac{\mathrm{i} \Omega_{2, n} p_{2} M(v)}{2\left[\gamma^{\prime}+\mathrm{i}\left(\delta_{2}-n \varpi+n \kappa v+k v\right)\right]} \\
& -\sum_{m} \frac{\mathrm{i} \Omega_{1, m+n} \mathrm{e}^{\mathrm{i} \varphi_{m}+\mathrm{i} \varphi_{n}-\mathrm{i} \varphi_{m+n}} r_{12, m}}{2\left[\gamma^{\prime}+\mathrm{i}\left(\delta_{2}-n \varpi+n \kappa v+k v\right)\right]} .
\end{aligned}
$$

Substituting the expressions for $\sigma_{13, n}$ and $\sigma_{32, n}$ from equation (18) into equations (15)(17) and taking into account equations (19), (20), we obtain the equation for $r_{12, n}$ which 
reads

$$
\begin{aligned}
& -\frac{M(v)}{4} \sum_{m}\left(\frac{p_{1} \Omega_{2, m} \Omega_{1, m+n} \mathrm{e}^{-\mathrm{i} \varphi_{m}-\mathrm{i} \varphi_{n}+\mathrm{i} \varphi_{m+n}}}{\gamma^{\prime}-\mathrm{i}\left[\delta_{1}-(m+n) \varpi+k v\right]}\right. \\
& \left.+\frac{p_{2} \Omega_{1, m} \Omega_{2, m-n} \mathrm{e}^{\mathrm{i} \varphi_{m}-\mathrm{i} \varphi_{n}-\mathrm{i} \varphi_{m-n}}}{\gamma^{\prime}+\mathrm{i}\left[\delta_{2}-(m-n) \varpi+k v\right]}\right) \\
& +\mathrm{i}\left(\omega_{21}-n \varpi\right) r_{12, n}-\gamma_{c o h} r_{12, n} \\
& -\nu\left[r_{12, n}-M(v) R_{12, n}\right] \\
& -\frac{1}{4} \sum_{m, l, j}\left(\frac{\Omega_{1, n+l} \Omega_{1, j+l} \mathrm{e}^{\mathrm{i} \varphi_{n+l}-\mathrm{i} \varphi_{n}+\mathrm{i} \varphi_{j}-\mathrm{i} \varphi_{j+l}}}{\gamma^{\prime}+\mathrm{i}\left(\delta_{2}-l \varpi+k v\right)}\right. \\
& \left.+\frac{\Omega_{2, l-n} \Omega_{2, l-j} \mathrm{e}^{-\mathrm{i} \varphi_{l-n}-\mathrm{i} \varphi_{n}+\mathrm{i} \varphi_{j}+\mathrm{i} \varphi_{l-j}}}{\gamma^{\prime}+\mathrm{i}\left(\delta_{1}-l \varpi+k v\right)}\right) r_{12, j}=0 .
\end{aligned}
$$

Here we neglected the terms with $\kappa$ in denominators owing to the condition $\kappa v / \varpi=$ $v / c \ll 1$ and introduced

$$
R_{12, n}=\int r_{12, n} d v
$$

We also neglected the coordinate derivative calculating $\frac{\mathrm{d}}{\mathrm{d} t} \rho_{12}$. Therefore, we neglect the Doppler broadening of the two-photon transition $|1\rangle \leftrightarrow|2\rangle$. It means that we assume $\frac{\omega_{21}}{c} v_{0}<\gamma_{c o l}$.

If the solution of equation (21) is known, it is possible to obtain the optical coherences and the atomic fluorescence intensity. In the next section we obtain the expression for the fluorescence intensity describing the dark resonance.

\section{Registered signal}

Observing the dark resonance in the polychromatic laser field, one registers the dependence of fluorescence intensity on the frequency difference $\varpi$ between adjacent spectral components. The fluorescence intensity is proportional to the number $S$ (hereinafter called the signal) of atoms excited by the laser field into the state $|3\rangle$ per unit of time,

$$
\begin{aligned}
S & =i \sum_{n} \int\left[\Omega_{1, n}\left(\rho_{31}-\rho_{13}\right)+\Omega_{2, n}\left(\rho_{32}-\rho_{23}\right)\right] \\
& \times \cos \left(k_{n} z-\omega_{n} t-\varphi_{n}\right) \mathrm{d} v .
\end{aligned}
$$

Averaging the expression (23) over time, we obtain the expression for the registered signal,

$$
\langle S\rangle=\sum_{n} \int \operatorname{Im}\left(r_{13, n} \Omega_{1, n}-r_{32, n} \Omega_{2, n}\right) \mathrm{d} v .
$$

Note that this result does not depend on the coordinate.

When we substitute equations (19) and (20) into equation (24), the contribution of

the summands which does not contain sums is proportional to the square of the laser 
field amplitude. This contribution (determined by $\gamma^{\prime}$ and Doppler width $k v_{0}$ of the optical transition) is practically constant in the vicinity of the CPT resonance. A part $\tilde{S}$ of the registered signal determined by the CPT resonance can be obtained by the substitution of the terms of equations (19) and (20) containing $r_{12, m}$ into equation (24),

$$
\begin{aligned}
\tilde{S} & =\operatorname{Re} \int \sum_{m, n}\left(\frac{\Omega_{1, n} \Omega_{2, n-m} \mathrm{e}^{\mathrm{i} \varphi_{m}-\mathrm{i} \varphi_{n}+\mathrm{i} \varphi_{n-m}}}{2\left[\gamma^{\prime}-\mathrm{i}\left(\delta_{1}-n \varpi+k v\right)\right]}\right. \\
& \left.+\frac{\Omega_{1, n} \Omega_{2, n-m} \mathrm{e}^{\mathrm{i} \varphi_{m}-\mathrm{i} \varphi_{n}+\mathrm{i} \varphi_{n-m}}}{2\left[\gamma^{\prime}+\mathrm{i}\left(\delta_{2}-(n-m) \varpi+k v\right)\right]}\right) r_{12, m} \mathrm{~d} v .
\end{aligned}
$$

In the next sections we consider the signal $\tilde{S}$ for different relations between the optical coherence relaxation rate $\gamma^{\prime}$, the Doppler width $k v_{0}$, the frequency $\omega_{21}$ of the transition between the two long-lived states, and the width $n_{0} \varpi$ of the FSF laser radiation spectrum.

\section{Large optical coherence relaxation rate}

When the optical coherence relaxation rate is larger than the Doppler width

$$
\gamma^{\prime} \gg k v_{0}
$$

we can neglect the velocity containing terms in denominators of (21) and (25) and perform the integration over velocity. Integration of the signal (25) gives the expression for the signal that contains $R_{12, m}$. The equations for $R_{12, m}$ can be found by integration of equation (21) over velocity. It is important that after this integration, equation (21) does not depend on the coherence preserving collision rate $\nu$. So, we conclude that the amplitude and the shape of the dark resonance do not depend on $\nu$ if inequality (26) is fulfilled. Below we consider the cases of "broad-band" and "narrow-band" (in comparison with $\gamma^{\prime}$ ) spectrum of laser radiation.

\subsection{The narrow-band spectrum of laser radiation}

In this subsection we consider the case when the expression (25) for the signal takes an especially simple form. Let us suppose that the optical coherence relaxation rate $\gamma^{\prime}$ is large in comparison with the laser spectrum width $n_{0} \varpi$ and with the detuning $\delta_{1}$ of the laser spectral component of maximal intensity from the transition frequency between states $|1\rangle$ and $|3\rangle$,

$$
\gamma^{\prime} \gg n_{0} \varpi, \quad \gamma^{\prime} \gg\left|\delta_{1}\right|
$$

As a result of the first inequality in (27), an inequality $\gamma^{\prime} \gg \omega_{21}$ is also valid.

It is evident from the structure of equations (21) that all $r_{12, n}$ are small in comparison with $r_{12, \tilde{m}}$, where $\tilde{m}$ is determined from the condition of $\left|\omega_{21}-\tilde{m} \varpi\right|$ minimum. Taking into account only the summand with $j=\tilde{m}$ in equation for $r_{12, \tilde{m}}$ we see that if the conditions (26) and (27) are fulfilled, the coefficient at $r_{12, \tilde{m}}$ in the 
second sum of equation (21) is real. Therefore, the second sum in equation (21) describes the field broadening. This broadening is small in comparison with $\gamma_{c o h}$ if

$$
\gamma^{\prime} \gamma_{c o h} \gg n_{0}\left(\Omega_{1,0}^{2}+\Omega_{2,0}^{2}\right) \text {. }
$$

In this subsection we assume that the inequality (28) is valid and, therefore, we neglect the field broadening. Then, from the equations (21) and (25), we obtain

$$
\begin{aligned}
\tilde{S} & =-\operatorname{Re} \sum_{n, m, l} \frac{\Omega_{1, n} \Omega_{2, n-m} \Omega_{1, l} \Omega_{2, l-m}}{4 \gamma^{\prime 2}\left[\gamma_{c o h}-\mathrm{i}(\delta+(\tilde{m}-m) \varpi)\right]} \\
& \times \mathrm{e}^{-\mathrm{i} \varphi_{n}+\mathrm{i} \varphi_{n-m}+\mathrm{i} \varphi_{l}-\mathrm{i} \varphi_{l-m}},
\end{aligned}
$$

where the two-photon detuning

$$
\delta=\omega_{21}-\tilde{m} \varpi
$$

is introduced. The main contribution into the sum over $m$ in the expression (29) comes from summands with $m=\tilde{m}$ because the frequency difference $\varpi$ between adjacent spectral components is much larger than the relaxation rate $\gamma_{c o h}$ of the coherence between $|1\rangle$ and $|2\rangle$ states. An example of the dependence of the signal (29) at twophoton resonance $(\delta=0)$ on the parameter $\alpha$ determining the spectral field component phases is presented in figure 2. One can see that the signal is maximal when $\alpha$ is divisible by $\pi / \tilde{m}$. These values of $\alpha$ can be also obtained from the expression for the linear combination of the phases in the exponent in the expression (29)

$$
\varphi_{l}-\varphi_{n}+\varphi_{n-m}-\varphi_{l-m}=2 \alpha m(l-n) .
$$

The right hand side of the equation (31) is divisible by $2 \pi$ for the summands with $m=\tilde{m}$ (which give the main contribution into the signal) and with any $l$ and $n$, if $\alpha=\alpha_{j}$ where

$$
\alpha_{j}=\frac{\pi}{\tilde{m}} j, \quad j \text { is integer. }
$$

Therefore, the contributions of the terms with different $n$ and $l$ are summed. If the difference between $\alpha$ and $\alpha_{j}$ is of order of

$$
\Delta \alpha=1 /\left(m n_{0}\right),
$$

the part of summands in expression (29) has different signs, and the signal magnitude decreases. Figure 2 shows that the equation (33) is in good accordance with the numerical simulation result: if $\left|\alpha-\alpha_{j}\right| \gtrsim 2 \Delta \alpha$ the signal practically vanishes.

This effect can be explained qualitatively in a different way. The scheme of the interaction between an atom and the laser field shown in figure 1 can be considered as a set of $\Lambda$-systems. We will call the $\Lambda$-system formed by $n$-th and $p$-th laser field component acting at $|1\rangle \leftrightarrow|3\rangle$ and $|2\rangle \leftrightarrow 3\rangle$ transition respectively as $\Lambda_{n, p}$. For each $\Lambda_{n, p^{-}}$-system, the dark state, i.e. the coherent superposition of states $|1\rangle$ and $|2\rangle$ that does not interact with $n$-th and $p$-th components of laser radiation, exists. However, these dark states depend on the relative phase $\varphi_{n, p}=\varphi_{n}-\varphi_{p}$ of laser components acting on the arms of $\Lambda_{n, p^{-}}$system. From the equation (3) we can see that $\varphi_{n, n-m}=2 \alpha m n-\alpha m^{2}$. If the frequency difference $\varpi$ between adjacent laser components becomes larger than the 


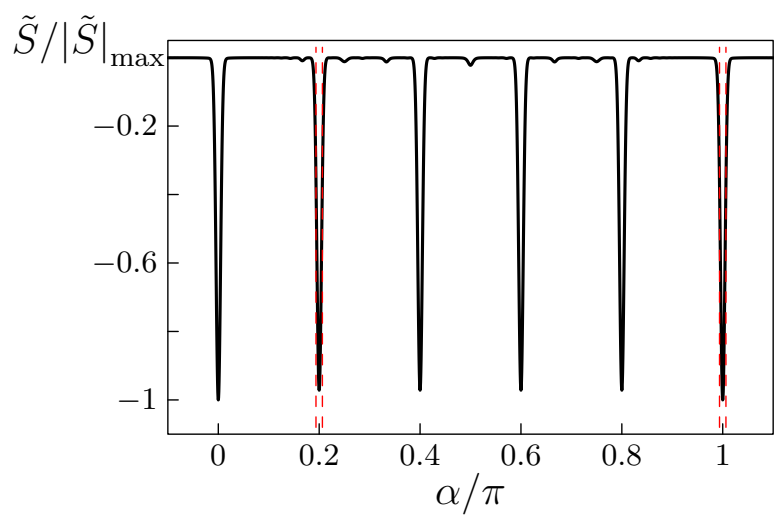

Figure 2. The normalized signal at two-photon resonance $(\delta=0)$ versus $\alpha$ for $n_{0}=10$, $\tilde{m}=5, \varpi=10 \gamma_{c o h}$. Dashed line corresponds to the difference $\Delta \alpha$ between $\alpha$ and $\alpha_{1}$

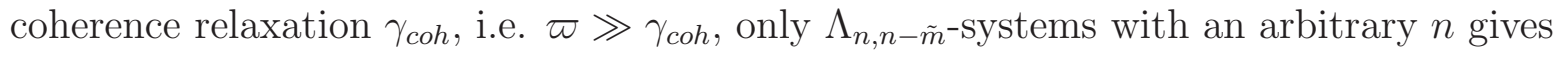
the contribution into the dark resonance. The dark states of $\Lambda_{n, n-\tilde{m}^{-}}$and $\Lambda_{l, l-\tilde{m}^{-} \text {-systems }}$ coincide when $\varphi_{n, n-\tilde{m}}-\varphi_{l, l-\tilde{m}}=2 \alpha \tilde{m}(n-l)$ is divisible by $2 \pi$ and therefore interference between them is constructive. This condition is fulfilled for any numbers of $n$ and $l$ only when $\alpha=\alpha_{j}$. If the condition $\left|\alpha-\alpha_{j}\right|>\Delta \alpha$ is fulfilled for any $j$, the destructive interference between various $\Lambda$-systems (the "dark state" for one $\Lambda$-system occurs to be the most light absorbing state for another $\Lambda$-system, etc.) leads to the considerable decreasing of the dark resonance amplitude. It is the interference of the different $\Lambda$ schemes contribution to the CPT resonance signal of an atom in a polychromatic field that distinguish behavior of the CPT resonances in the polychromatic and bichromatic fields.

Figure 3 shows the time dependence of the FSF laser radiation intensity for $\alpha$ close to $\alpha_{1}$ and $\alpha_{\tilde{m}}=\pi$. As one can see from figure 3 $a$, if $\alpha=\alpha_{1}$, the laser radiation field is a sequence of light pulses that follow each other with a period of $T / 5$, where $T=2 \pi / \varpi$. Variation of $\alpha$ on $\Delta \alpha$ leads to more than twofold decrease of the amplitude and to the considerable broadening of the pulses. Another change of $\alpha$ on $\Delta \alpha$ leads to the distortion of the periodical light pulse sequence. In the case of $\alpha=\pi$ (figure $3 b$ ) the laser field consists of the sequence of light pulses with the repetition period $T$. Increasing of $|\alpha-\pi|$ leads to decreasing of the pulse amplitude and to the broadening of pulses. Therefore, the optimal conditions for the dark resonance formation are satisfied when the time dependence of the laser radiation is the periodical sequence of the light pulses of the maximal amplitude. Both values of $\alpha$ corresponding to pulses of the maximal amplitudes are the particular cases of the special lasing regimes in which the FSF laser emits a train of the short pulses [26]. In general case, the laser emits such pulses when $\alpha=\pi n / m$ where $n$ and $m$ are integer.

If $\alpha$ is close to the optimal values $\alpha_{j}$, the dependence of the signal on the two-photon detuning is well described by a Lorentzian curve with a HWHM $\gamma_{c o h}$. Summands with 
Dark resonances in the field of frequency shifted feedback laser radiation

$(a)$

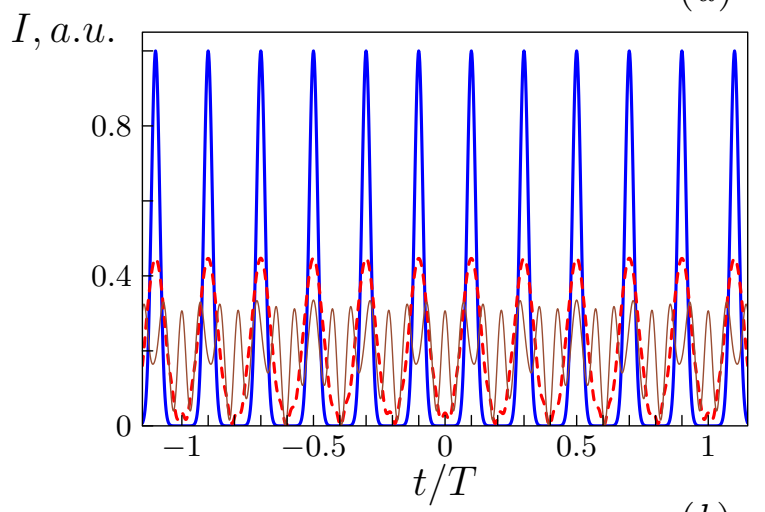

$(b)$

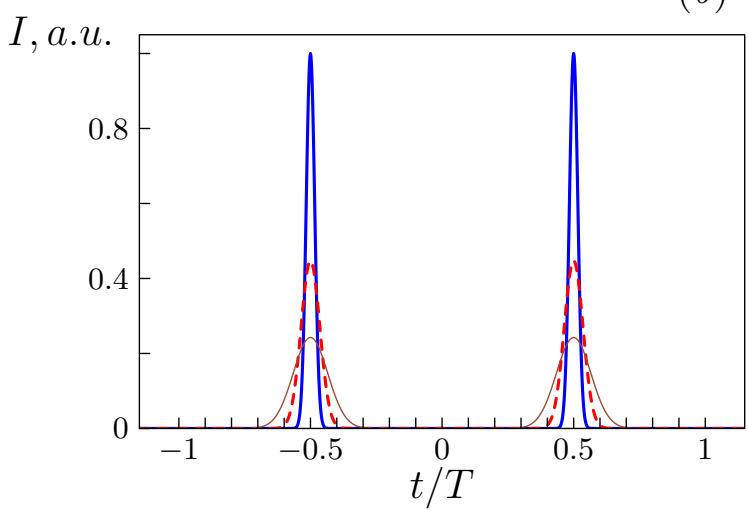

Figure 3. Time dependence of the laser field intensity in arbitrary units for $n_{0}=10$. a) $\alpha=\alpha_{1}$ (thick solid line), $\alpha=\alpha_{1}+\Delta \alpha$ (dashed line), $\alpha=\alpha_{1}+2 \Delta \alpha$ (thin solid line); b) $\alpha=\pi$ (thick solid line), $\alpha=\pi+\Delta \alpha$ (dashed line), $\alpha=\pi+2 \Delta \alpha$ (thin solid line)

$m \neq \tilde{m}$ lead to the small shift $\delta_{s}$ of the signal minimum from the two-photon resonance

$$
\delta_{s}=-\left[\frac{\partial}{\partial \delta} \tilde{S}\left(\frac{\partial^{2}}{\partial \delta^{2}} \tilde{S}\right)^{-1}\right]_{\delta=0} .
$$

The dependence of the shift (34) on the difference $\alpha-\alpha_{j}$ for parameters corresponding to figure 2 is shown in figure 4. As one can see, $\left.\delta_{s}\right|_{\alpha=\pi}$ is approximately 40 times greater than $\left.\delta_{s}\right|_{\alpha=\pi / \tilde{m}}$. The reason for this difference is that at $\alpha=\pi$ the summands in the expression (29) have the same signs due to multiple of $2 \pi$ argument of cosine and sine functions whereas for $\alpha=\pi / \tilde{m}$ only the part of summands have the argument which is divisible by $2 \pi$. Other summands has different values of cosine and sine arguments and these values are distributed approximately homogeneously in the interval $[0,2 \pi]$ if $\tilde{m}$ is large. As a result, the sum of summands containing Lorentzians with $m \neq \tilde{m}$ is reduced. 
Dark resonances in the field of frequency shifted feedback laser radiation

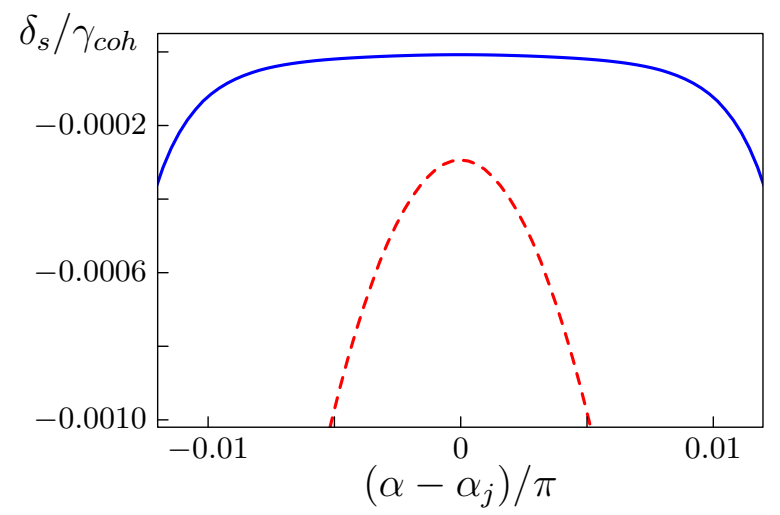

Figure 4. Dependence of the shift of the dark resonance minimum from the exact twophoton resonance on $\alpha-\alpha_{j}$ for $j=1$ (solid line) and $j=5$ (dashed line). Parameters are the same as for figure 2

\subsection{Broad-band spectrum of the laser radiation}

In this section we suppose that only the condition (26) is valid whereas the relations between the width of the laser spectrum, the frequency of $|1\rangle \leftrightarrow|2\rangle$ transition and the optical coherences relaxation rate $\gamma^{\prime}$ are arbitrary. In the previous subsection we established that the main contribution into the equation (21) and the signal (25) comes from summands with $r_{12, \tilde{m}}$, namely from the summands with $m=\tilde{m}$. These summands are responsible for the two-photon transitions between the long-lived states in the twophoton resonance conditions when the frequency difference between spectral components is close to the frequency of transition $|1\rangle \leftrightarrow|2\rangle$. The other summands result in the small shift of the CPT resonance position from the two-photon resonance. Neglecting this small shift, from the equations (21) and (25) we obtain

$$
\begin{aligned}
\tilde{S} & =\operatorname{Re} \sum_{n}\left(\frac{\Omega_{1, n} \Omega_{2, n-\tilde{m}} \mathrm{e}^{\mathrm{i} \varphi_{\tilde{m}}-\mathrm{i} \varphi_{n}+\mathrm{i} \varphi_{n-\tilde{m}}}}{2\left[\gamma^{\prime}-\mathrm{i}\left(\delta_{1}-n \varpi\right)\right]}\right. \\
& \left.+\frac{\Omega_{1, n} \Omega_{2, n-\tilde{m}} \mathrm{e}^{\mathrm{i} \varphi_{\tilde{m}}-\mathrm{i} \varphi_{n}+\mathrm{i} \varphi_{n-\tilde{m}}}}{2\left[\gamma^{\prime}+\mathrm{i}\left(\delta_{2}-(n-\tilde{m}) \varpi\right)\right]}\right) R_{12, \tilde{m}} .
\end{aligned}
$$

where

$$
\begin{aligned}
& R_{12, \tilde{m}}=-\frac{1}{4\left[\gamma_{c o h}+\gamma_{b}-\mathrm{i}\left(\delta-\delta_{f}\right)\right]} \\
& \times \sum_{m}\left(\frac{p_{1} \Omega_{1, m} \Omega_{2, m-\tilde{m}} \mathrm{e}^{\mathrm{i} \varphi_{m}-\mathrm{i} \varphi_{\tilde{m}}-\mathrm{i} \varphi_{m-\tilde{m}}}}{\gamma^{\prime}-\mathrm{i}\left[\delta_{1}-m \varpi\right]}\right. \\
& \left.+\frac{p_{2} \Omega_{1, m} \Omega_{2, m-\tilde{m}} \mathrm{e}^{\mathrm{i} \varphi_{m}-\mathrm{i} \varphi_{\tilde{m}}-\mathrm{i} \varphi_{m-\tilde{m}}}}{\gamma^{\prime}+\mathrm{i}\left[\delta_{2}-(m-\tilde{m}) \varpi\right]}\right) .
\end{aligned}
$$


In the expression for $R_{12, \tilde{m}}, \gamma_{b}$ is the field broadening width and $\delta_{f}$ is the dark resonance light shift. They read

$$
\begin{aligned}
\gamma_{b}= & \frac{\gamma^{\prime}}{4} \sum_{l}\left(\frac{\Omega_{1, l}^{2}}{\gamma^{\prime 2}+\left(\delta_{1}-l \varpi\right)^{2}}\right. \\
& \left.+\frac{\Omega_{2, l}^{2}}{\gamma^{\prime 2}+\left(\delta_{2}-l \varpi\right)^{2}}\right), \\
\delta_{f}= & \frac{1}{4} \sum_{l}\left(\frac{\Omega_{1, l}^{2}\left(\delta_{1}-l \varpi\right)}{\gamma^{\prime 2}+\left(\delta_{1}-l \varpi\right)^{2}}\right. \\
& \left.-\frac{\Omega_{2, l}^{2}\left(\delta_{2}-l \varpi\right)}{\gamma^{\prime 2}+\left(\delta_{2}-l \varpi\right)^{2}}\right) .
\end{aligned}
$$

It is easy to see from the equations (35) and (36) that if $\alpha$ is determined by the expression (32), the contributions with different values of $n, m$ into the signal are summed, and the signal is maximal. If $\alpha$ differs from the optimal values defined by expression (32), the signal decreases due to the presence of the summands with different phases.

If $\gamma^{\prime} \gg \varpi$ and $n_{0} \gg 1$, the summation in the expression (38) for the light shift can be replaced by the integration over $l$. The analytical expression of the integral for the Gaussian spectral distribution of intensity cannot be obtained. For estimations we replace the Gaussian spectral distribution by the Lorentzian distribution

$$
\Omega_{j, l}^{2}=\Omega_{j, 0}^{2}\left(1+\frac{2 \pi l^{2}}{n_{0}^{2}}\right)^{-1} .
$$

Replacing summation by integration in the expression (38) and taking into account the expression (39) we find

$$
\delta_{f}=\Delta_{1}-\Delta_{2},
$$

where

$$
\Delta_{j}=\frac{\Omega_{j, 0}^{2} n_{0} \delta_{j} \sqrt{\pi^{3}}\left[\left(\sqrt{2 \pi \sqrt{2}} \gamma^{\prime}-n_{0} \varpi 2^{1 / 4}\right)^{2}+2 \pi \sqrt{2} \delta_{j}^{2}\right]}{4\left[4 \pi^{2}\left(\delta_{j}^{2}+\gamma^{\prime 2}\right)^{2}+n_{0}^{2} \varpi^{2}\left(n_{0}^{2} \varpi^{2}+4 \pi \delta_{j}^{2}-4 \pi \gamma^{\prime 2}\right)\right]} .
$$

Figure 5 shows examples of the light shift dependencies on the detuning $\delta_{1}$ of the laser radiation spectrum maximum from the frequency of transition $|1\rangle \leftrightarrow|3\rangle$ calculated using the expressions (38) and (40). One can see that the results calculated using both formulae are in good correspondence when $\delta_{1}$ is small (of order of $\gamma^{\prime}$ ). This enables us to find from (40) the value of $\delta_{1}=\delta_{z}$ for which the light shifts caused by the field acting at $|1\rangle \leftrightarrow|3\rangle$ and $|2\rangle \leftrightarrow|3\rangle$ transitions compensate one another. If the spectrum is broad-band $\left(n_{0} \varpi \gg \gamma^{\prime}\right)$ and if the dipole matrix elements of the transition $|1\rangle \leftrightarrow|3\rangle$, $|2\rangle \leftrightarrow|3\rangle$ are different, we find

$$
\delta_{z}=\frac{\omega_{21} \Omega_{2,0}^{2}}{\Omega_{2,0}^{2}-\Omega_{1,0}^{2}}=\frac{\omega_{21} d_{23}^{2}}{d_{23}^{2}-d_{13}^{2}} .
$$


Dark resonances in the field of frequency shifted feedback laser radiation

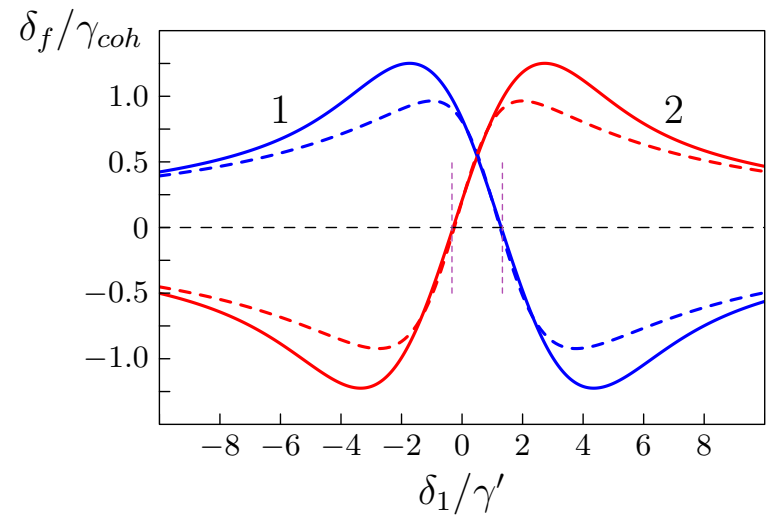

Figure 5. The dependence of the light shift on the detuning $\delta_{1}$ of the maximal intensity spectral component frequency from the frequency of transition between states $|1\rangle$ and $|3\rangle$. Parameters: $\gamma^{\prime}=\omega_{21}=1000 \gamma_{c o h}, \varpi=20 \gamma_{c o h}, n_{0}=400$. The Rabi frequencies corresponding to the maximal intensity spectral components are: $1-\Omega_{1,0}=5 \gamma_{c o h}, \Omega_{2,0}=10 \gamma_{c o h}, 2-\Omega_{1,0}=10 \gamma_{c o h}, \Omega_{2,0}=5 \gamma_{c o h}$. Solid line the expression (38) evaluation, dashed line - the expression (40) evaluation. The magnitudes of $\delta_{z}$ calculated using (42) are shown by vertical segments.

Here we used the equality $\Omega_{1,0} / \Omega_{2,0}=d_{13} / d_{23}$. Let us note that if $\delta_{1}=\delta_{z}$ and the parameters of the atom-field interaction correspond to figure 5, the magnitude of the signal is close to a maximum.

\section{Small optical coherence relaxation rate}

The case when $\gamma^{\prime}$ is small in comparison with the Doppler width of the spectrum,

$$
\gamma^{\prime} \leq k v_{0}
$$

we consider in the approximation (28) neglecting the field broadening and the light shifts. From the expressions (21) and (25) we find that

$$
\begin{aligned}
\tilde{S}= & -\frac{1}{8} \operatorname{Re} \sum_{n, m, l} \int \Omega_{1, l} \Omega_{2, l-m} \Omega_{1, n} \Omega_{2, n-m} \mathrm{e}^{\mathrm{i} \varphi_{l}-\mathrm{i} \varphi_{l-m}-\mathrm{i} \varphi_{n}+\mathrm{i} \varphi_{n-m}} \\
& \times\left(2 \gamma^{\prime}-\mathrm{i} \delta-\mathrm{i} \tilde{m} \varpi+\mathrm{i} m \varpi\right)\left[\gamma^{\prime}-\mathrm{i}\left(\delta_{1}-n \varpi+k v\right)\right]^{-1} \\
& \times\left[\gamma^{\prime}+\mathrm{i}\left(\delta_{1}-\delta-n \varpi+m \varpi-\tilde{m} \varpi+k v\right)\right]^{-1} \\
& \times\left(\frac{p_{2}}{\gamma^{\prime}+\mathrm{i}\left[\delta_{1}-\delta-(l+\tilde{m}-m) \varpi+k v\right]}\right. \\
& +\frac{\nu p_{2} F\left(\left[\delta_{1}-\delta-l \varpi+m \varpi-\tilde{m} \varpi\right] / \gamma^{\prime}\right)}{\gamma^{\prime}\left[\gamma_{c o h}-\mathrm{i}(\delta+\tilde{m} \varpi-m \varpi)\right]}+ \\
& \left.+\frac{p_{1}}{\gamma^{\prime}-\mathrm{i}\left[\delta_{1}-l \varpi+k v\right]}+\frac{\nu p_{1} F\left(\left[\delta_{1}-l \varpi\right] / \gamma^{\prime}\right)^{*}}{\gamma^{\prime}\left[\gamma_{c o h}-\mathrm{i}(\delta+\tilde{m} \varpi-m \varpi)\right]}\right) \\
& \times \frac{M(v)}{\gamma_{c o h}+\nu-\mathrm{i}(\delta+\tilde{m} \varpi-m \varpi)} d v .
\end{aligned}
$$




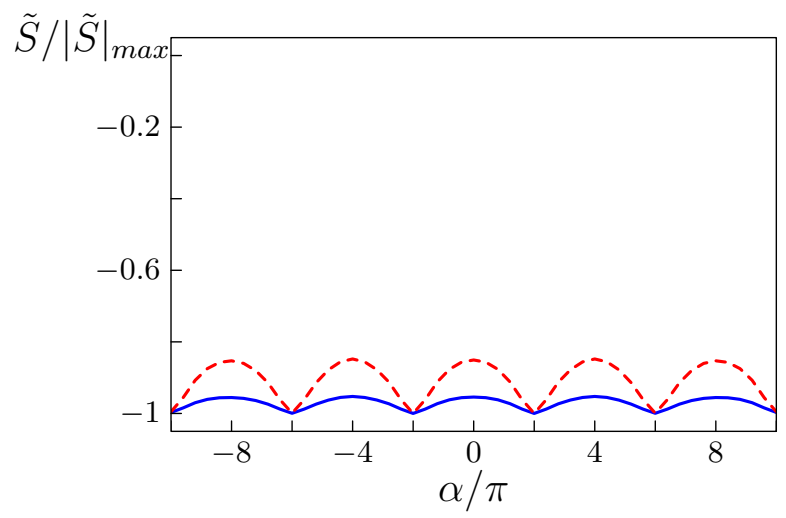

Figure 6. The dependence of the normalized signal at two-photon resonance $(\delta=0)$ on $\alpha$ for $n_{0}=5, \tilde{m}=5, \varpi=200 \gamma_{c o h}, p_{1}=p_{2}=0.5, k v_{0}=5000 \gamma_{c o h}, \nu=0$. Solid line corresponds to $\gamma^{\prime}=10 \gamma_{c o h}$, dashed line corresponds to $\gamma^{\prime}=20 \gamma_{c o h}$

Here we introduce the function of dimensionless variable $x$

$$
F(x)=\int_{-\infty}^{\infty} \frac{\gamma^{\prime} M(v)}{\gamma^{\prime}+\mathrm{i}\left(\gamma^{\prime} x+k v\right)} d v .
$$

If $\gamma^{\prime} \ll k v_{0}$ and $\gamma^{\prime} \ll \varpi$ but $\gamma^{\prime} \gg \gamma_{c o h}$, the main contribution into the signal (44) comes from the summands with $m=\tilde{m}, l=n$. Therefore the triple summation is reduced to a single one and the result of summing does not depend on the phases of the spectral components of the field. Figure [6 shows the weak, more weak than in figure 2, dependence of the signal at two-photon resonance on $\alpha$ that decreases with decreasing of $\gamma^{\prime}$.

In contrast to the case of large optical coherence relaxation rate $\gamma^{\prime} \gg k v_{0}$ considered above, when the signal does not depend on the rate $\nu$ of coherence preserving collisions, in the case $\gamma^{\prime} \leq k v_{0}$ the signal strongly depends on $\nu$. Examples of dependencies of the signal on $\delta$ for $\gamma^{\prime}>\varpi$ are shown in figure 7 . One can see that increasing of $\nu$ leads to the initial increasing and subsequent decreasing of the dark resonance HWHM. Figure 8 shows the dependence of the signal on the collision frequency if the condition of two-photon resonance is fulfilled. One can see that increasing of $\nu$ leads to decreasing of registered signal, fast initially and slow at $\nu \gg \gamma_{c o h}$.

This effect can be explained qualitatively in the following way. If $\gamma^{\prime} \gg k v$, the certain atom after each collision remains being interacting with the same components of laser radiation as before the collision, i.e., with all the components whose Dopplershifted frequencies $\omega_{n}-k v$ are close enough to the optical transition frequencies $\omega_{31}$ or $\omega_{32}$ in the atom, $\left|\omega_{n}-\omega_{31,32}-k v\right|<<\gamma^{\prime}$, therefore velocity changes due to collisions with the buffer gas atoms do not lead to any additional broadening or shift of the dark resonances. If $\gamma^{\prime} \leq k v$, any collision will modify the set of the laser components that interacts with the atom. Therefore the collisional rate $\nu$ occurs to be an important parameter determining the dark resonance properties. 


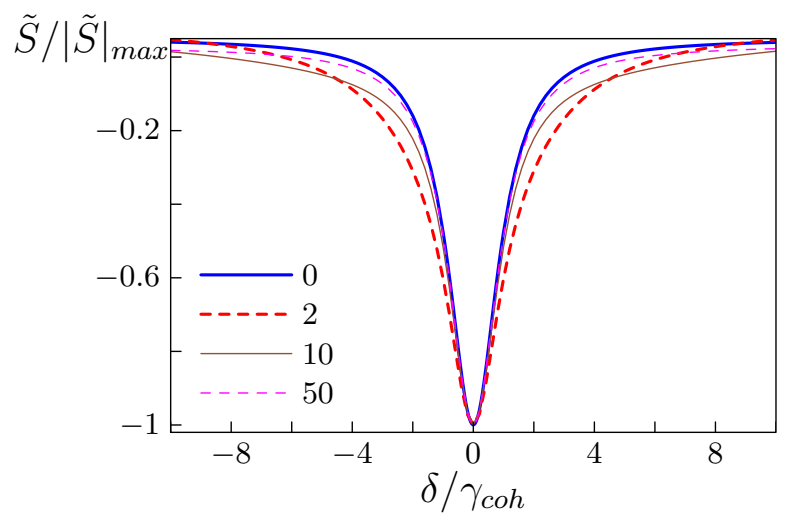

Figure 7. The dependence of the signal (normalized to unit magnitude at twophoton resonance) on the two-photon detuning for $n_{0}=10, \tilde{m}=10, \varpi=50 \gamma_{\text {coh }}$, $p_{1}=p_{2}=0.5, k v_{0}=2000 \gamma_{c o h}, \alpha=\pi, \gamma^{\prime}=200 \gamma_{c o h}$. The curves are marked by the magnitude of $\nu / \gamma_{c o h}$

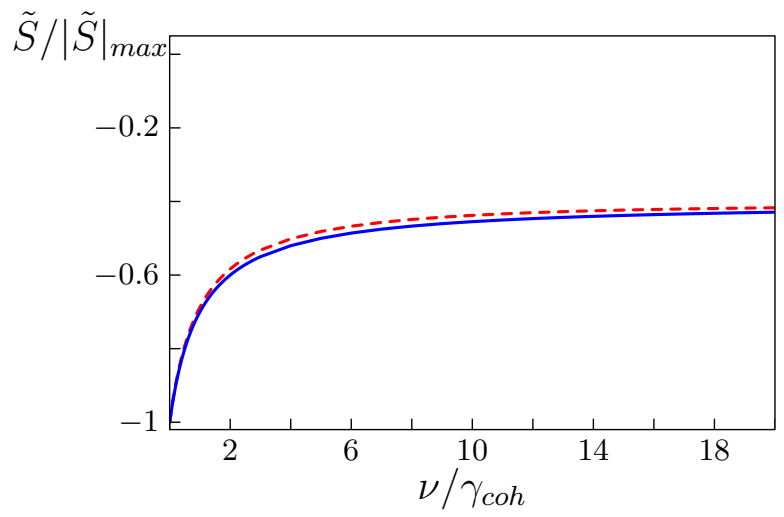

Figure 8. The dependence of the normalized signal on the collision preserving frequency at two-photon resonance condition $(\delta=0)$ for $n_{0}=10, \tilde{m}=10, \varpi=50 \gamma_{c o h}$, $p_{1}=p_{2}=0.5, k v_{0}=2000 \gamma_{c o h}, \gamma^{\prime}=200 \gamma_{c o h}$; solid line corresponds to $\alpha=\pi / \tilde{m}$, dashed line corresponds to $\alpha=\pi$

We should keep in mind that parameters $\gamma^{\prime}, \gamma_{c o h}$ and $\nu$ are not independent. While $\gamma^{\prime}$ and $\nu$ increase linearly with the buffer gas pressure, $\gamma_{c o h}$ decreases approximately as a square root of the pressure. Without buffer gas, $\gamma_{c o h}$ is approximately equal to the inverse time of flight of an atom through the laser beam, and in buffered cells it is approximately equal to the inverse time of diffusion of an atom out of the beam (this dependence occurs to be more complicated if an atom can return back to the laser beam without loss of the coherence, see $[29,30]$ ). When we consider the coherence preserving collisions rate as an independent parameter calculating the dependencies shown in figure 8 we just estimate its influence on the registered signal magnitude. 


\section{Conclusions}

We present the theory of the dark resonances in a fluorescence of a three-level atom gas interacting with a polychromatic field of a frequency shifted feedback (FSF) laser. We show that conditions for the resonance observation are optimal when the phase relations between the laser spectral components provide generation of a light pulses train. We study analytically the field broadening and the light shift of the resonances.

The expressions for the field broadening width and light shift of the dark resonances are found for the large (in comparison with Doppler width) optical coherence relaxation rate.

We found the condition on the detuning of the spectral component with maximal intensity from the transition frequency between one of the long-lived states and the short-lived state that provides zero value of the light shift. This condition does not depend on the laser field strength.

It is shown that increasing of the coherence preserving collisions rate leads to decreasing of the signal. This decreasing is quick at small collision rate and becomes more slow with increasing of the collision rate.

\section{Acknowledgments}

The work was financially supported by State Foundation of Fundamental Researches of Ukraine (project F28.2/035), Russian Foundation for Basic Researches (project 0902-90465_Ukr_f_a), FTsP "Scientific and Pedagogical personnel of innovative Russia" (project P2326 from 16.11.2009), Russian President Grant for Young Candidates of Sciences (project MK-5318.2010.2).

\section{References}

[1] Arimondo E and Orriols G 1976 Nuovo Cimento Lett. 17 333-338

[2] Alzetta G, Gozzini A, Moi L and Orriols G 1976 Nuovo Cimento B Ser. 2 36 5-20

[3] Gray H R, Whitley R W and Stroud C R Jr 1978 Optics Lett. 3 218-220

[4] Orriols G 1979 Nuovo Cimento B Ser. 253 1-24

[5] Alzetta G, Moi L and Orriols G 1979 Nuovo Cimento B Ser. 252 209-218

[6] Kocharovskaya O A and Khanin Ya I 1986 Sov. Phys. JETP 63 945-950

[7] Gornyı̆ M B, Matisov B G and Rozhdestvenskiü Yu V 1989 Sov. Phys. JETP 68 728-732

[8] Boller K-J, Imamoglu A and Harris S E 1991 Phys. Rev. Lett. 66 2593-2596

[9] Harris S E 1997 Phys. Today 50 36-42

[10] Oreg J, Hioe F T and Eberly J H 1984 Phys. Rev. A 29 690-697

[11] Gaubatz U, Rudecki P, Becker M, Schiemann S, Kulz M and Bergmann K 1988 Chem. Phys. Lett. $149463-468$

[12] Bergmann K, Theur H and Shore B W 1998 Rev. Mod. Phys. 70 1003-1025

[13] Vitanov N V, Halfmann T, Shore B W, Bergmann K 2001 Annu. Rev. Phys. Chem. 52 763-809

[14] Aspect A, Arimondo E, Kaiser R, Vansteenkiste N and Cohen-Tannoudji C 1988 Phys. Rev. Lett. $61826-829$

[15] Knappe S, Wynands R, Kitching J, Robinson H G and Hollberg L 2001 J. Opt. Soc. Am. B 18 $1545-1553$ 
[16] Nagel A, Graf L, Naumov A, Mariotti E, Biancalana V, Meschede D and Wynands R 1998 Europhys. Lett. 44 31-36

[17] Schwindt P D D, Knappe S, Shah V, Hollberg L, Kitching J, Liew L-A and Moreland J 2004 Appl. Phys. Lett. 85 6409-6411

[18] Dicke R H 1953 Phys. Rev. 89 472-473

[19] Erhard M and Helm H 2001 Phys. Rev. A 63043814

[20] Merimaa M, Lindvall T, Tittonen I and Ikonen E 2003 J. Opt. Soc. Am. B 20 273-279

[21] Alzetta G, Gozzini S, Lucchesini A, Cartaleva S, Karaulanov T, Marinelli C and Moi L 2004 Phys. Rev. A 69, 063815

[22] Sautenkov V A, Rostovtsev Yu V, Ye C Y, Welch G R, Kocharovskaya O and Scully M O 2005 Phys. Rev. A 71063804

[23] Arissian L and Diels J-C 2006 Optics Communications 264 169-173

[24] Vladimirova Yu V, Grishanin B A, Zadkov V N, Biancalana V, Bevilacqua G, Dancheva Y and Moi L 2006 JETP 103 528-538

[25] Auzinsh M, Malitskiy R A, Matsnev I V, Negriyko A M, Romanenko V I and Yatsenko L P 2009 Ukr. J. Phys. 54 974-982

[26] Yatsenko L P, Shore B W and Bergmann K 2004 Optics Communications 236 183-202

[27] Rautian S G 1967 Sov. Phys. JETP 24 788-796

[28] Rautian S G and Sobel'man I I 1967 Sov. Phys. Usp. 9 701-716

[29] Xiao Y, Novikova I, Phillips D F, Walsworth R L 2006 Phys. Rev. Lett. 96043601

[30] Romanenko V I, Romanenko A V, Yatsenko L P 2010 Ukr. J. Phys. 55 393-402 